MATHEMATICS OF COMPUTATION

Volume 75, Number 255, July 2006, Pages 1067-1085

S 0025-5718(06)01823-0

Article electronically published on March 3, 2006

\title{
POINTWISE ERROR ESTIMATES FOR DISCONTINUOUS GALERKIN METHODS WITH LIFTING OPERATORS FOR ELLIPTIC PROBLEMS
}

\author{
JOHNNY GUZMÁN
}

\begin{abstract}
In this article, we prove some weighted pointwise estimates for three discontinuous Galerkin methods with lifting operators appearing in their corresponding bilinear forms. We consider a Dirichlet problem with a general second-order elliptic operator.
\end{abstract}

\section{INTRODUCTION}

Discontinuous Galerkin (DG) methods for elliptic problems have received considerable attention in the last few years. A unified analysis of $L_{2}$-based global estimates was given by Arnold et. al. 22 for nine DG methods. In that article, in order to do the unified analysis, they cast all the methods in their primal forms (although some methods are more natural in their mixed forms). Four of the methods were shown to be consistent, to be adjoint consistent and to have coercive bilinear forms for the Laplacian. With these properties, they were able to show optimal convergence rates for the gradient and function values. For these four methods, a natural question arises: How do these methods behave pointwise? Kanschat and Rannacher 8 ] gave a quasi-optimal convergence result in $L_{\infty}$ for the interior penalty (IP) method, and Chen and Chen [6] gave weighted pointwise estimates for the same method, which implies the result in [8]. In this paper, we show weighted pointwise error estimates for the three remaining methods.

One main difference between the IP method and the three methods considered here is that the latter have terms with lifting operators appearing in their bilinear forms. As pointed out in 2], the IP method can be problematic since the penalty parameters must be chosen sufficiently large to make the method stable. The three remaining methods do not have this problem.

Once one has local $H^{1}$ estimates, weighted pointwise estimates are easily obtained following the pointwise estimates proof of Schatz [12] for the standard continuous Galerkin method or a similar proof in [6] for the IP method. Therefore, our main contribution is to prove local $H^{1}$ estimates for these methods. The local $H^{1}$ analysis becomes more difficult because of the presence of lifting operators. Here we define one of the lifting operators. Let $e$ be the edge shared by triangles $T_{1}$ and

Received by the editor June 27, 2004 and, in revised form, April 19, 2005.

2000 Mathematics Subject Classification. Primary 65N30, 65N15.

Key words and phrases. Finite elements, discontinuous Galerkin.

The author was supported by a Ford Foundation Fellowship and a Cornell-Sloan Fellowship.

(C)2006 American Mathematical Society

Reverts to public domain 28 years from publication 1067 
$T_{2}$, and let $q \in\left[L_{2}(e)\right]^{2}$. We define $r_{M, e}:\left[L^{2}(e)\right]^{2} \rightarrow\left[V_{h}\right]^{2}$ by the following identity:

$$
\int_{T_{1} \cup T_{2}} r_{M, e}(q)^{T} M \tau d x=-\int_{e} q^{T}\langle M \tau\rangle d s, \quad \forall \tau \in\left[V_{h}\right]^{2} .
$$

Here $M$ is a symmetric, uniformly positive definite matrix, $\langle\cdot\rangle$ is the average operator across $e$ and $V_{h}$ denotes our subspace of discontinuous functions. One of the difficulties that we overcame is determining proper bounds for terms of the form $\left\|\omega^{2} r_{M, e}\left(\left[u_{h}\right]\right)-r\left(\omega^{2}\left[u_{h}\right]\right)\right\|_{L_{2}\left(T_{1} \cup T_{2}\right)}$, where $\omega$ is a cut-off function and $\left[u_{h}\right]$ denotes the jump of our approximation across the edge $e$. In order to do this, we use $L_{2}$ type projection operators and a modification of super-approximation (see Lemmas 2.2 and 2.3 .

Chen [5] proved some local $H^{1}$ error estimates for the local discontinuous Galerkin (LDG) method in its mixed formulation. In this paper, we do the analysis for the LDG method in its primal form, and we repeat the analysis for two other methods. Lifting operators do not appear in the mixed formulation for the LDG method. Therefore, using the mixed formulation avoids the difficulties of analyzing lifting operators. However, one cannot avoid these difficulties in the remaining two methods, because lifting operators also appear in their mixed formulations.

The pointwise estimates obtained here and in [6] are modeled on the pointwise estimates obtained for the standard continuous Galerkin method in [12]. Let $V_{h}$ be the space of discontinuous functions such that the restriction of a function to an element is a polynomial of degree $r-1$. The pointwise estimates take on the following forms (compare to Theorems 2.1, 3.1 in [12] and Theorems 5.1, 5.2 in [6]):

$$
\left|\left(u-u_{h}\right)(x)\right| \leq C h \inf _{\chi \in V_{h}}|||u-\chi| \|_{W_{h}^{1, \infty}(\Omega), x, s}, \quad 0 \leq s<r-2
$$

and

$$
\left|\nabla_{h}\left(u-u_{h}\right)(x)\right| \leq C \inf _{\chi \in V_{h}} \mid\|u-\chi\|_{W_{h}^{1, \infty}(\Omega), x, s}, \quad 0 \leq s<r-1 .
$$

Here, $\nabla_{h} \phi$ denotes the piecewise defined function such that $\nabla_{h} \phi=\nabla \phi$ on each element of the triangulation. The weighted norm appearing on the right-hand sides of (1.1), (1.2) are precisely defined in Section 2.3, and it will be clear that we can bound that norm by the weighted norm defined in [12] if $\chi$ is continuous. More precisely, $\left.|||u-\chi|\right|_{W_{h}^{1, \infty}(\Omega), x, s} \leq C\left(\left\|\sigma_{x}^{s}(u-\chi)\right\|_{L_{\infty}(\Omega)}+\left\|\sigma_{x}^{s} \nabla(u-\chi)\right\|_{L_{\infty}(\Omega)}\right)$, where $\sigma_{x}(y)=h /(|x-y|+h)$. Therefore, if $s=0$ (no weight) we get estimates in the $L_{\infty}$-norm. However, if $s>0$ our error will be localized around $x$. Consequently, we can also show expansion inequalities ([12]) for these DG methods. The inequalities (1.1), (1.2) will hold if $s=r-2$ and $s=r-1$, respectively, as long as we add a logarithmic factor to the right-hand side of the inequalities (see Theorems 4.1, 4.2).

The rest of this paper is organized as follows: In the next section, we present some preliminaries. We define the problem in a precise way, and we introduce our bilinear forms. Then, in Section 2.4 we develop some important approximation results. We end the preliminaries by proving some estimates for lifting operators and by bounding the bilinear forms. In Section 3, we prove local $H^{1}$ estimates. Finally, in Section 4, we state our pointwise estimates. 


\section{Preliminaries}

2.1. Dirichlet problem. Let $\Omega \subset R^{2}$ be bounded with smooth boundary. We consider the following Dirichlet problem:

$$
\begin{aligned}
L u \equiv-\nabla \cdot(A(x) \nabla u)+b(x) \cdot \nabla u+c(x) u & =f & & \text { in } \Omega, \\
u & =0 & & \text { on } \partial \Omega .
\end{aligned}
$$

The components of $A=\left(a_{i j}\right)_{1 \leq i, j \leq 2}, b=\left(b_{i}\right)_{1 \leq i \leq 2}$ and $c$ are assumed to be smooth and bounded. Furthermore, we assume that $A$ is symmetric and uniformly positive definite in $\Omega$. That is, there exists a constant $C_{\text {ell }}>0$ such that

$$
\psi^{T} A(x) \psi \geq C_{\text {ell }}|\psi|^{2}, \quad \forall x \in \Omega \text { and } \psi \in R^{2} .
$$

We assume that (2.1) has a unique solution in $H_{0}^{1}(\Omega)$ for all $f \in L_{2}(\Omega)$.

In this paper we are not going to trace the dependence of constants on the ellipticity factor $C_{e l l}$ and upper bounds for $A(x), b(x)$ or $c(x)$.

2.2. Discontinuous approximating spaces and bilinear forms. Suppose we have a family of triangulations $\mathcal{T}_{h}$ (possibly with hanging nodes) that fit $\Omega$ exactly, where $\Omega=\bigcup_{T \in \mathcal{T}_{h}} T$. Let $h=\sup _{T \in \mathcal{T}_{h}} h_{T}$, where $h_{T}=\operatorname{diam}(T)$. Let $V_{h, p}$ denote the finite-dimensional space of functions that are polynomials of degree at most $p$ on each element, and define $\Sigma_{h, p}=V_{h, p} \times V_{h, p}$. From now on, we set $V_{h}=V_{h, r-1}$ and $\Sigma_{h}=\Sigma_{h, r-1}$. We naturally define the collection of interior edges as $\mathcal{E}_{h}^{0}=\left\{\partial T \cap \partial T^{\prime}\right.$ : $T, T^{\prime} \in \mathcal{T}_{h}, T \neq T^{\prime}$, meas $\left._{1}\left(\partial T \cap \partial T^{\prime}\right) \neq 0\right\}$ and the collection of boundary edges, in general curved, as $\mathcal{E}_{h}^{\partial}=\left\{\partial T \cap \partial \Omega: T \in \mathcal{T}_{h}\right.$, meas $\left._{1}(\partial T \cap \partial \Omega) \neq 0\right\}$. The collection of all edges will be denoted by $\mathcal{E}_{h}=\mathcal{E}_{h}^{\partial} \cup \mathcal{E}_{h}^{0}$. $S_{e}$ will denote the union of elements that have $e$ as an edge. We assume that our elements are non-degenerate; that is, there exists a constant $C_{n d}>0$, independent of $T$, such that $h_{T} \leq C_{n d} \operatorname{diam}\left(B_{T}\right)$, where $B_{T}$ is the largest ball contained in $T$. Furthermore, we assume the existence of a constant $C_{E}>0$, independent of $h$ and $e$, with $h \leq C_{E} h_{e}$, where $h_{e}=\operatorname{length}(e)$. This is the quasi-uniform condition that was used in $[6$.

We say that $T$ and $T^{\prime}$ are neighbors if $\partial T \cap \partial T^{\prime} \in \mathcal{E}_{h}^{0}$. From the quasi-uniform condition, it follows that there exists a positive integer $K$ independent of $h$ such that each $T \in \mathcal{T}_{h}$ has at most $K$ neighbors (if our meshes do not have hanging nodes, then $K$ can be 3 ).

On each edge, as in 2], we define the average and jump operators as follows for $e \in \mathcal{E}_{h}^{0}$ :

$$
\begin{array}{ll}
\langle q\rangle=\frac{1}{2}\left(q_{1}+q_{2}\right), & {[q]=q_{1} \cdot n_{1}+q_{2} \cdot n_{2},} \\
\langle\phi\rangle=\frac{1}{2}\left(\phi_{1}+\phi_{2}\right), & {[\phi]=\phi_{1} n_{1}+\phi_{2} n_{2},}
\end{array}
$$

for $q$ vector valued and $\phi$ scalar valued. Here $S_{e}=T_{1} \cup T_{2}, q_{i}=\left.q\right|_{T_{i}}, \phi_{i}=\left.\phi\right|_{T_{i}}$, and $n_{i}$ is the exterior normal to $T_{i}, i=1,2$. For $e \in \mathcal{E}_{h}^{\partial}$,

$$
\langle q\rangle=q, \quad[\phi]=\phi n,
$$

where $n$ is the outward unit normal. Note that $[q]$ is a scalar and $[\phi]$ a vector. The quantities $[q]$ and $\langle\phi\rangle$ on boundary edges are not required, so they are left undefined. 
Now we present some local lifting operators as in 2]. Let $M$ be a symmetric, smooth, bounded and uniformly positive definite matrix in $\Omega$. Let $r_{M, e}:\left[L^{2}(e)\right]^{2} \rightarrow$ $\Sigma_{h}$ and $l_{M, e}: L^{2}(e) \rightarrow \Sigma_{h}$ be given by

$$
\begin{aligned}
\int_{S_{e}} r_{M, e}(q)^{T} M \tau d x & =-\int_{e} q^{T}\langle M \tau\rangle d s, \\
\int_{S_{e}} l_{M, e}(\phi)^{T} M \tau d x & =-\int_{e} \phi[M \tau] d s, \quad \forall \tau \in \Sigma_{h} .
\end{aligned}
$$

We set the global lifting operators to be $r_{M}(q)=\sum_{e \in \mathcal{E}_{h}} r_{M, e}(q)$ and $l_{M}(\phi)=$ $\sum_{e \in \mathcal{E}_{h}^{0}} l_{M, e}(\phi)$.

Now, we are ready to define the bilinear forms. They are the modified BRMPS 3], modified BMMPR 4 and local discontinuous Galerkin bilinear forms. (See [2] for the bilinear forms for the Laplacian.) The following term is common to all three:

$$
\begin{aligned}
\theta(u, v)= & \sum_{T \in \mathcal{T}_{h}} \int_{T}\left(\nabla u^{T} A \nabla v+\left(b^{T} \nabla u\right) v+c u v\right) d x \\
& -\sum_{e \in \mathcal{E}_{h}} \int_{e}\left(\left\langle A \nabla_{h} u\right\rangle[v]+\left\langle A \nabla_{h} v\right\rangle[u]+b^{T}[u]\langle v\rangle\right) d s .
\end{aligned}
$$

\section{Modified BRMPS}

$$
B(u, v)=\theta(u, v)+\sum_{e \in \mathcal{E}_{h}} \eta_{e} \int_{S_{e}} r_{A, e}([u])^{T} A r_{A, e}([v]) d x+\sum_{e \in \mathcal{E}_{h}^{\partial}} \frac{1}{h_{e}} \int_{e}[u][v] d s .
$$

\section{Modified BMMPR}

$$
\begin{aligned}
B(u, v)= & \theta(u, v)+\int_{\Omega} r_{A}([u])^{T} A r_{A}([v]) d x \\
& +\sum_{e \in \mathcal{E}_{h}} \eta_{e} \int_{S_{e}} r_{I, e}([u])^{T} r_{I, e}([v]) d x+\sum_{e \in \mathcal{E}_{h}^{\partial}} \frac{1}{h_{e}} \int_{e}[u][v] d s .
\end{aligned}
$$

\section{Local Discontinuous Galerkin}

$$
\begin{aligned}
B(u, v)= & \theta(u, v)+\int_{\Omega}\left(r_{A}([u])+l_{A}\left(\beta^{T}[u]\right)\right)^{T} A\left(r_{A}([v])+l_{A}\left(\beta^{T}[v]\right)\right) d x \\
& -\sum_{e \in \mathcal{E}_{h}^{0}} \int_{e}\left(\left[A \nabla_{h} u\right] \beta^{T}[v]+\beta^{T}[u]\left[A \nabla_{h} v\right]\right) d s+\sum_{e \in \mathcal{E}_{h}} \frac{\eta_{e}}{h_{e}} \int_{e}[u][v] d s .
\end{aligned}
$$

Here $\eta_{e}$ is constant for each $e$, and is bounded for all $e$. If we let $\eta=\inf _{e \in \mathcal{E}_{h}} \eta_{e}$, then we require that $K<\eta$ (See Section 2.2 for the definition of $K$ ) for the Modified Bassi form and $0<\eta$ for the two remaining forms. Also, $\beta$ is a constant vector on each interior edge and is bounded component-wise for all $e$. Our modification of the first two methods is solely motivated by our analysis. It consists of adding the last terms over the boundary edges. Without this modification we were not able to prove Theorem 3.1 since we were unable to show (2.19) for curved edges. Whether this modification is necessary in practice we do not know. We intend to investigate this question in the future.

The discontinuous approximation $u_{h}$ solves

$$
B\left(u_{h}, v\right)=(f, v), \quad \forall v \in V_{h} .
$$

For each method we use the corresponding bilinear form. 
2.3. Discontinuous Sobolev norms. If $D \subset \Omega$, we define our discontinuous Sobolev space as in [6]:

$$
W_{h}^{l, p}(D)=\left\{v: v \in W^{l, p}(T \cap D), \forall T \in \mathcal{T}_{h}\right\} .
$$

This space is equipped with the norm $(1 \leq p<\infty)$

$$
\|v\|_{W_{h}^{l, p}(D)}=\left(\sum_{j=0}^{l}|v|_{W_{h}^{j, p}(D)}^{p}\right)^{\frac{1}{p}},|v|_{W_{h}^{j, p}(D)}=\left(\sum_{T \in \mathcal{T}_{h}}|v|_{W^{j, p}(T \cap D)}^{p}\right)^{\frac{1}{p}},
$$

with the appropriate modification for $p=\infty$. When $p=2$, we set $H_{h}^{l}(D)=$ $W_{h}^{l, p}(D)$.

Let $D \subset \Omega$ and $1 \leq p<\infty$. The norms that occur in most of our analysis take on the following form:

$$
\begin{aligned}
\|v\| \|_{W_{h}^{l, p}(D)}^{p}= & \|v\|_{W_{h}^{l, p}(D)}^{p}+\sum_{e \in \mathcal{E}_{h}} h_{e}^{1-p}\|[v]\|_{L_{p}(e \cap D)}^{p} \\
& +\sum_{e \in \mathcal{E}_{h}} h_{e}\left\|\left\langle\nabla_{h} v\right\rangle\right\|_{L_{p}(e \cap D)}^{p}+\sum_{e \in \mathcal{E}_{h}^{0}} h_{e}\left\|\left[\nabla_{h} v\right]\right\|_{L_{p}(e \cap D)}^{p} .
\end{aligned}
$$

For $p=\infty$,

$$
\begin{aligned}
\|v\| \|_{W_{h}^{l, \infty}(D)}= & \|v\|_{W_{h}^{l, \infty}(D)}+\sup _{e \in \mathcal{E}_{h}} \frac{1}{h_{e}}\|[v]\|_{L_{\infty}(e \cap D)} \\
& +\sup _{e \in \mathcal{E}_{h}}\left\|\left\langle\nabla_{h} v\right\rangle\right\|_{L_{\infty}(e \cap D)}+\sup _{e \in \mathcal{E}_{h}^{0}}\left\|\left[\nabla_{h} v\right]\right\|_{L_{\infty}(e \cap D) .}
\end{aligned}
$$

We consider the weight $\sigma_{x}^{s}(y)=\left(\frac{h}{|x-y|+h}\right)^{s}$ as in [12]. If we let

$$
\|v\|_{W_{h}^{l, p}(\Omega), x, s}^{p}=\left\|\sigma_{x}^{s} v\right\|_{L_{p}(\Omega)}^{p}+\left\|\sigma_{x}^{s} \nabla_{h} v\right\|_{L_{p}(\Omega)}^{p}
$$

for $1 \leq p<\infty$, then for fixed $x$ we define the norms

$$
\begin{aligned}
\|\| v\|\|_{W_{h}^{l, p}(\Omega), x, s}^{p}= & \|v\|_{W_{h}^{l, p}(\Omega), x, s}^{p}+\sum_{e \in \mathcal{E}_{h}} h_{e}^{1-p}\left\|\sigma_{x}^{s}[v]\right\|_{L_{p}(e)}^{p} \\
& +\sum_{e \in \mathcal{E}_{h}} h_{e}\left\|\sigma_{x}^{s}\left\langle\nabla_{h} v\right\rangle\right\|_{L_{p}(e)}^{p}+\sum_{e \in \mathcal{E}_{h}^{0}} h_{e}\left\|\sigma_{x}^{s}\left[\nabla_{h} v\right]\right\|_{L_{p}(e)}^{p},
\end{aligned}
$$

again with the appropriate modification for $p=\infty$.

2.4. Approximation. We start by stating well-known trace inequalities. Let $e$ be an edge of $T \in \mathcal{T}_{h}$ and $\phi$ be either a scalar- or vector-valued function. Then, for $1 \leq p \leq \infty$, we have

$$
\|\phi\|_{L_{p}(e)} \leq C\left(h^{-\frac{1}{p}}\|\phi\|_{L_{p}(T)}+h^{1-\frac{1}{p}}|\phi|_{W^{1, p}(T)}\right) .
$$

If we restrict $\phi$ to $V_{h, i}$ or $\Sigma_{h, i}$, for some fixed $i>0$, we can state some inverse inequalities that are also well known:

$$
\begin{gathered}
\|\phi\|_{W^{l, t}(T)} \leq C h^{\left[\frac{2}{l}-\frac{2}{q}\right]+s-t}\|\phi\|_{W^{q, s}(T)}, \\
\|\phi\|_{L_{p}(e)} \leq C h^{-\frac{1}{p}}\|\phi\|_{L_{p}(T)},
\end{gathered}
$$

where $C$ does not depend on $\phi, h, e$, or $T$.

The following is a standard elementwise approximation result. Let

$$
v \in W_{h}^{j, p}(\Omega)\left(\left[W_{h}^{j, p}(\Omega)\right]^{2}\right)
$$


with $0 \leq i \leq j \leq r$. Then, there exists a $\phi \in V_{h}\left(\Sigma_{h}\right)$ with

$$
\|v-\phi\|_{W^{i, p}(T)} \leq C h^{j-i}|v|_{W^{j, p}(T)}, \quad \forall T \in \mathcal{T}_{h},
$$

where $C$ does not depend on $v, h$, or $T$.

We present some function spaces, as in [13, that will help us in stating further approximation results. If $S \subset R \subset \Omega$, let $\partial_{<}(S, R)=\operatorname{dist}(\partial S \backslash \partial \Omega, \partial R \backslash \partial \Omega)$. The spaces are defined as follows:

$$
V_{h}^{<}(A)=\left\{v \in V_{h}: \partial_{<}(\operatorname{supp}(v), A)>0\right\},
$$

and

$$
C_{<}^{\infty}(A)=\left\{v \in C^{\infty}: \partial_{<}(\operatorname{supp}(v), A)>0\right\} .
$$

The following lemma follows from trace inequalities and elementwise approximation. (See section 4.3 in [2] for a similar result.)

Lemma 2.1. Let $D_{0} \subset D_{d}$ with $\partial_{<}\left(D_{0}, D_{d}\right)=d \geq \kappa h$ (for a fixed $\kappa>1$ sufficiently large). Let $v \in H_{h}^{r}\left(D_{d}\right)$. Then, there exists $\psi \in V_{h}$ such that

$$
\left.|||v-\psi|\right|_{H_{h}^{1}\left(D_{0}\right)} \leq C h^{r-1}|v|_{H_{h}^{r}\left(D_{d}\right)},
$$

where $C$ is independent of $v, h, D_{0}$ and $D_{d}$. Furthermore, if $\operatorname{supp}(v) \subset D_{0}$, then $\psi \in V_{h}^{<}\left(D_{d}\right)$.

Throughout this paper, we are going to be estimating functions of the form $v=\omega \chi$ or $v=\omega^{2} \chi$, where $\chi \in V_{h}$ or $\Sigma_{h}$ and $\omega$ is a cut-off function. Hence, we develop some approximation results for these functions.

Lemma 2.2. Let $\chi \in V_{h}$, and let $\omega$ be a smooth function. Suppose there exist constants $C>0$ and $d \geq \kappa h$ for some constant $\kappa>1$ such that $|\omega|_{W^{l, \infty}(\Omega)} \leq C d^{-l}$ for $l=0,1, \ldots, r+1$. Then,

$$
\begin{gathered}
\left|\omega^{2} \chi\right|_{H^{r}(T)} \leq C \frac{1}{h^{r-2}}\left(d^{-1}|\omega \chi|_{H^{1}(T)}+d^{-2}\|\chi\|_{L_{2}(T)}\right), \\
\left|\omega^{2} \chi\right|_{H^{r}(T)} \leq C \frac{1}{h^{r-2}}\left(d^{-1} h^{-1}\|\omega \chi\|_{L_{2}(T)}+d^{-2}\|\chi\|_{L_{2}(T)}\right), \\
|\omega \nabla(\omega \chi)|_{H^{r}(T)} \leq C \frac{1}{h^{r-1}}\left(d^{-1}\|\nabla(\omega \chi)\|_{L_{2}(T)}+d^{-2}\|\chi\|_{L_{2}(T)}\right), \\
|\omega \chi|_{H^{k}(T)} \leq C \frac{1}{h^{k-1}}\left(|\omega \chi|_{H^{1}(T)}+d^{-1}\|\chi\|_{L_{2}(T)}\right) \quad \text { for } k=1, \ldots, r-1,
\end{gathered}
$$

and

$$
|\omega \chi|_{H^{r}(T)} \leq C \frac{1}{h^{r-1}} d^{-1}\|\chi\|_{L_{2}(T)} .
$$

Here $C$ is independent of $\omega, \chi, T$, and $h$.

Proof. By Leibniz's rule, the fact that the $r$ th derivatives of $\chi$ vanish in $T$ and inverse estimates, we get that

$$
\left.\left|\omega^{2} \chi\right|_{H^{r}(T)} \leq C\left(\sum_{l=2}^{r} h^{l-r}\left|\omega^{2}\right|_{W^{l, \infty}(T)}\right)\|\chi\|_{L_{2}(T)}+\sum_{|\alpha|=1,|\beta|=r-1}\left\|D^{\alpha} \omega^{2} D^{\beta} \chi\right\|_{L_{2}(T)}\right) .
$$

By the decay properties of $\omega$ and the fact that $h d^{-1}<1$, we see that

$$
\left(\sum_{l=2}^{r} h^{l-r}\left|\omega^{2}\right|_{W^{l, \infty}(T)}\right)\|\chi\|_{L_{2}(T)} \leq C d^{-2} h^{2-r}\|\chi\|_{L_{2}(T)} .
$$


Now we handle the second sum in (2.13). Note that $D^{\alpha} \omega^{2}=2 \omega D^{\alpha} \omega$ since $|\alpha|=1$. Therefore,

$$
\sum_{|\alpha|=1,|\beta|=r-1}\left\|D^{\alpha} \omega^{2} D^{\beta} \chi\right\|_{L_{2}(T)} \leq C d^{-1} \sum_{|\beta|=r-1}\left\|\omega D^{\beta} \chi\right\|_{L_{2}(T)} .
$$

Now we let $\hat{\omega}=\frac{1}{|T|} \int_{T} \omega d x$. By the triangle inequality, we have that

$$
\left\|\omega D^{\beta} \chi\right\|_{L_{2}(T)} \leq\left\|(\omega-\hat{\omega}) D^{\beta} \chi\right\|_{L_{2}(T)}+\left\|\hat{\omega} D^{\beta} \chi\right\|_{L_{2}(T)} .
$$

Using approximation properties, we see that

$$
\left\|(\omega-\hat{\omega}) D^{\beta} \chi\right\|_{L_{2}(T)} \leq\left. C h|\omega|_{W^{1, \infty}(T)}|| D^{\beta} \chi\right|_{L_{2}(T)} .
$$

Using inverse estimates and decay properties of $\omega$, we have

$$
\left\|(\omega-\hat{\omega}) D^{\beta} \chi\right\|_{L_{2}(T)} \leq C d^{-1} h^{2-r}\|\chi\|_{L_{2}(T)} .
$$

To handle the next term we again use an inverse estimate to get

$$
\left\|\hat{\omega} D^{\beta} \chi\right\|_{L_{2}(T)} \leq C h^{2-r}|\hat{\omega} \chi|_{H^{1}(T)} .
$$

Using the triangle inequality, we have

$$
|\hat{\omega} \chi|_{H^{1}(T)} \leq|(\hat{\omega}-\omega) \chi|_{H^{1}(T)}+|\omega \chi|_{H^{1}(T)} .
$$

By using the product rule, approximation properties, and inverse estimates, we obtain

Therefore,

$$
|(\hat{\omega}-\omega) \chi|_{H^{1}(T)} \leq C d^{-1}\|\chi\|_{L_{2}(T)} .
$$

$$
|\hat{\omega} \chi|_{H^{1}(T)} \leq C\left(\left.d^{-1}|| \chi\right|_{L_{2}(T)}+|\omega \chi|_{H^{1}(T)}\right) .
$$

Combining these estimates, we have that

$$
\sum_{|\alpha|=1,|\beta|=r-1}\left\|D^{\alpha} \omega^{2} D^{\beta} \chi\right\|_{L_{2}(T)} \leq d^{-1} h^{2-r}|\omega \chi|_{H^{1}(T)}+d^{-2} h^{2-r}|| \chi \|_{L_{2}(T)} .
$$

This proves (2.8). By again introducing $\hat{\omega}$ we can bound $|\omega \chi|_{H^{1}(T)}$ by the righthand side of (2.9). This will prove (2.9). By using the triangle inequality we see that $|\omega \nabla(\omega \chi)|_{H^{r}(T)} \leq\left|\omega^{2} \nabla(\chi)\right|_{H^{r}(T)}+|\omega \chi \nabla \omega|_{H^{r}(T)}$. We can then use (2.9) to bound $\left|\omega^{2} \nabla(\chi)\right|_{H^{r}(T)}$ by the right-hand side of (2.10), and we can use Leibniz's rule and inverse estimates to bound $|\omega \chi \nabla \omega|_{H^{r}(T)}$. This would prove (2.10). By Leibniz's rule, inverse estimates and using the decay properties of $\omega$, we can prove (2.11) and (2.12). We omit the proofs.

Now we can state a super-approximation result similar to that in 12. The differences between this approximation result and the one contained in [12] is that $\omega$ appears on the right-hand side of our result. This will allow us to perform "kick back" arguments. (See the proof of Theorem 3.1)

Lemma 2.3. Let $\partial_{<}\left(D_{0}, D_{d}\right)=d>\kappa h$, where $\omega \in C_{<}^{\infty}\left(D_{0}\right)$. Suppose

$$
|\omega|_{W^{l, \infty}\left(D_{0}\right)} \leq C d^{-l}
$$

for $l=0,1, \ldots, r+1$. Then, for all $\chi \in V_{h}$ there exists $\psi \in V_{h}^{<}\left(D_{d}\right)$ with

$$
\left\||| \omega^{2} \chi-\psi \mid\right\|_{H_{h}^{1}(\Omega)} \leq C h\left(d^{-1}|\omega \chi|_{H_{h}^{1}\left(D_{d}\right)}+d^{-2}\|\chi\|_{L_{2}\left(D_{d}\right)}\right),
$$

where $C$ is independent of $\chi$ and $\omega$.

Proof. This easily follows from Lemma 2.1 and (2.8). 
2.5. Boundedness and consistency of the forms. We start by defining and stating some properties of certain projection operators that we use throughout this paper. In this direction, let $M$ be a symmetric, smooth, bounded matrix that is uniformly positive definite in $\Omega$. If $g \in L_{2}(\Omega)$, we define $P_{M}(g) \in \Sigma_{h}$ by the following equation:

$$
\int_{\Omega} \chi^{T} M g=\int_{\Omega} \chi^{T} M P_{M}(g), \quad \forall \chi \in \Sigma_{h} .
$$

Note that $P_{M}$ can be defined elementwise since $\Sigma_{h}$ is a space of discontinuous functions. If $M=I$, then this will simply be the vector-valued $L_{2}$-projection operator. $L_{2}$-projection operators have been analyzed extensively. The proof of the following lemma, which we omit, follows the proof for the $L_{2}$-projection operator ([7); however, it is much simpler since $V_{h}$ consists of discontinuous functions.

Lemma 2.4. Let $M$ be a symmetric, smooth and bounded matrix that is uniformly positive definite. Let $P_{M}$ be defined by (2.14) and $1 \leq p \leq \infty$. Then, for all $T \in \mathcal{T}_{h}$,

$$
\left\|P_{M}(g)\right\|_{L_{p}(T)} \leq C_{1}\|g\|_{L_{p}(T)}
$$

and

$$
\| g-\left.P_{M}(g)\right|_{H^{k}(T)} \leq C_{2} h^{r-k}|g|_{H^{r}(T)} \text { for } k=1, \ldots, r,
$$

where $C_{1}$ and $C_{2}$ are independent of $g$ and $T$.

In order to show boundedness of our forms, we need some estimates for our lifting operators. The following lemma is an extension of Lemma 2(ii) in [4].

Lemma 2.5. Let $M$ be smooth, bounded, and uniformly positive definite in $\Omega$. Let $1 \leq p, q \leq \infty, \frac{1}{p}+\frac{1}{q}=1$. Then, there exists a constant $C$ such that for all $\phi \in\left[L_{p}\left(e_{1}\right)\right]^{2}$ and $\chi \in\left[L_{q}\left(e_{2}\right)\right]^{2}$,

$$
\begin{aligned}
\left|\int_{S_{e_{1}} \cap S_{e_{2}}} r_{M, e_{1}}(\phi)^{T} M r_{M, e_{2}}(\chi) d x\right| & +\left|\int_{S_{e_{1}} \cap S_{e_{2}}} l_{M, e_{1}}(\phi)^{T} M r_{M, e_{2}}(\chi) d x\right| \\
& +\left|\int_{S_{e_{1}} \cap S_{e_{2}}} l_{M, e_{1}}(\phi)^{T} M l_{M, e_{2}}(\chi)\right| d x \mid \\
\leq & C \frac{1}{h}\|\phi\|_{L_{p}\left(e_{1}\right)}|| \chi \|_{L_{q}\left(e_{2}\right)}
\end{aligned}
$$

In the case that $e_{1}$ and $e_{2}$ do not belong to the same triangle, the left-hand side of (2.15) will be zero.

Proof. The last statement of the lemma follows by the definition of the lifting operators. In the other case, by the boundedness of $M$ and (2.5), we have

$$
\begin{aligned}
\left|\int_{S_{e_{1}} \cap S_{e_{2}}} r_{M, e_{1}}(\phi)^{T} M r_{M, e_{2}}(\chi) d x\right| & =\left|\int_{e_{1}} \phi^{T} M\left\langle r_{M, e_{2}}(\chi)\right\rangle d s\right| \\
& \leq C\|\phi\|_{L_{p}\left(e_{1}\right)}\left\|\left\langle r_{M, e_{2}}(\chi)\right\rangle\right\|_{L_{q}\left(e_{1}\right)} \\
& \leq C h^{-\frac{1}{q}}\|\phi\|\left\|_{L_{p}\left(e_{1}\right)}\right\| r_{M, e_{2}}(\chi) \|_{L_{q}\left(S_{e_{2}}\right)} .
\end{aligned}
$$

Now we use a duality argument to bound $\left\|r_{M, e_{2}}(\chi)\right\|_{L_{q}\left(S_{e_{2}}\right)}$. If $g=M^{-1} z$, then

$$
\int_{S_{e_{2}}} r_{M, e_{2}}(\chi)^{T} z d x=-\int_{e_{2}} \chi^{T} M\left\langle P_{M}(g)\right\rangle d s
$$


By Hölder's inequality, (2.5), and Lemma 2.4 we easily get

$$
-\int_{e_{2}} \chi^{T} M\left\langle P_{M}(g)\right\rangle d s \leq C h^{-\frac{1}{p}}\|\chi\|_{L_{q}\left(e_{2}\right)} \mid\|g\|_{L_{p}\left(S_{e_{2}}\right)} .
$$

Therefore, by duality $\left\|r_{M, e_{2}}(\chi)\right\|_{L_{q}\left(S_{e_{2}}\right)} \leq C h^{-\frac{1}{p}}\|\chi\|_{L_{q}\left(e_{2}\right)}$ since $\|g\|_{L_{p}\left(S_{e_{2}}\right)} \leq$ $C\|z\|_{L_{p}\left(S_{e_{2}}\right)}$. Hence, we have established the following:

$$
\left|\int_{S_{e_{1}} \cap S_{e_{2}}} r_{M, e_{1}}(\phi)^{T} M r_{M, e_{2}}(\chi) d x\right| \leq C \frac{1}{h}\|\phi\|_{L_{p}\left(e_{1}\right)}\|\chi\|_{L_{q}\left(e_{2}\right)} .
$$

The last two terms can be bounded following similar steps.

In the case that $e_{1}$ and $e_{2}$ belong to a common triangle, a simple exercise shows $\max _{y \in e_{1}}\left(\sigma_{x}^{s}(y)\right) \leq 2^{s} \min _{y \in e_{2}}\left(\sigma_{x}^{s}(y)\right)$. Therefore,

$$
\frac{1}{h}\|\phi\|_{L_{p}\left(e_{1}\right)}\|\chi\|_{L_{q}\left(e_{2}\right)} \leq C \frac{1}{h}\left\|\sigma^{-s} \phi\right\|_{L_{p}\left(e_{1}\right)}\left\|\sigma^{s} \chi\right\|_{L_{q}\left(e_{2}\right)} .
$$

If we take into account that $r_{M}=\sum_{e \in \mathcal{E}_{h}} r_{M, e}, l_{M}=\sum_{e \in \mathcal{E}_{h}^{0}} l_{M, e}$, make use of Hölder's inequality (both for the integrals and summation), and apply Lemma 2.5 and (2.17), we can show the following boundedness of our forms. (See [6], 2] for analogous results.)

Lemma 2.6. Let $1 \leq p, q \leq \infty, \frac{1}{p}+\frac{1}{q}=1$. For all three forms, there exists a positive constant $C$ such that for all $u \in W_{h}^{1, p}(\Omega)$ and $v \in W_{h}^{1, q}(\Omega)$

$$
|B(v, u)|+|B(u, v)| \leq\left. C|||u|\right|_{W_{h}^{1, p}(\Omega), x, s}|||v| \|_{W_{h}^{1, q}(\Omega), x,-s},
$$

where $C$ is independent of $x, u$ and $v$.

The next important inequality is an extension of Lemma 2(i) in [4].

Lemma 2.7. Let $M$ be given as in Lemma 2.5. Let $e \in \mathcal{E}_{h}^{0}, \phi \in\left[P_{r-1}(e)\right]^{2}$ and suppose $\omega \in C^{\infty}(\Omega)$. Then,

$h_{e}^{-1} \int_{e}(\omega \phi)^{T} M(\omega \phi) d s \leq C\left(\int_{S_{e}} \omega^{2} r_{M, e}(\phi)^{T} M r_{M, e}(\phi) d x+h_{e}|\omega|_{W^{1, \infty}\left(S_{e}\right)}^{2}\|\phi\|_{L_{2}(e)}^{2}\right)$.

Proof. Let $v \in\left[P_{p}(e)\right]^{2}$ for some fixed $p$. Then, $v$ is defined naturally on the line containing $e$, call it $l$. As in [4, we define $\bar{v} \in\left[P_{p}\left(R^{2}\right)\right]^{2}$ as the vector-valued polynomial satisfying $v=\bar{v}$ on $l$ and which is constant on the lines perpendicular to $l$. As pointed out in [4,

$$
\|\bar{v}\|_{L_{2}\left(S_{e}\right)}^{2} \leq C h_{e}\|v\|_{L_{2}(e)}^{2} .
$$

Also, let $\tilde{\omega} \equiv \min _{\bar{S}_{e}} \omega$. It follows by the mean value theorem, possibly applying it twice, that

$$
\| \tilde{\omega}-\left.\omega\right|_{L_{\infty}\left(S_{e}\right)} \leq C h|\omega|_{W^{1, \infty}\left(S_{e}\right)} .
$$

We easily see by our definitions that

$$
\int_{e}(\omega \phi)^{T} M(\omega \phi) d s=E_{1}+E_{2}
$$


where

$$
\begin{aligned}
& E_{1}=h_{e}^{-1} \int_{e}\left((\omega \phi)^{T} M(\omega \phi)-(\tilde{\omega} \phi)^{T} M(\tilde{\omega} \phi)\right) d s, \\
& E_{2}=-h_{e}^{-1} \tilde{\omega}^{2} \int_{S_{e}}(\bar{\phi})^{T} M r_{M, e}(\phi) d s .
\end{aligned}
$$

We rewrite $E_{1}$ as

$$
E_{1}=C h_{e}^{-1} \int_{e}(\omega-\tilde{\omega})(\omega+\tilde{\omega}) \phi^{T} M \phi d s
$$

By the Cauchy-Schwarz inequality and the arithmetic-geometric mean inequality we have

$$
E_{1} \leq C h_{e}^{-1} \int_{e}(\omega-\tilde{\omega})^{2} \phi^{T} M \phi d s+\epsilon h_{e}^{-1} \int_{e}(\omega+\tilde{\omega})^{2} \phi^{T} M \phi d s .
$$

Later we will choose $\epsilon>0$ sufficiently small. Finally, using (2.21) and $(\omega+\tilde{\omega})^{2} \leq$ $2\left(\omega^{2}+\tilde{\omega}^{2}\right) \leq 2 \omega^{2}$, we see that

$$
E_{1} \leq C h_{e}|\omega|_{W^{1, \infty}\left(S_{e}\right)}^{2}\|\phi\|_{L_{2}(e)}^{2}+2 \epsilon h^{-1} \int_{e} \omega^{2} \phi^{T} M \phi d s .
$$

Again, using the Cauchy-Schwarz inequality and the arithmetic-geometric mean inequality, we have

$$
E_{2} \leq \tilde{\omega}^{2}\left(\epsilon h^{-2} \int_{S_{e}} \bar{\phi}^{T} M \bar{\phi} d x+C \int_{S_{e}} r_{M, e}(\phi)^{T} M r_{M, e}(\phi) d x\right) .
$$

By using (2.20) and the positive definiteness of $M$, we see that

$$
E_{2} \leq C_{1} \epsilon \int_{e} \omega^{2} \phi^{T} M \phi d s+C \int_{S_{e}} \omega^{2} r_{M, e}(\phi)^{T} M r_{M, e}(\phi) d x,
$$

where $C_{1}$ is a constant independent of $\epsilon$.

Finally, taking $\epsilon$ small enough, we arrive at our conclusion.

The next lemma can easily be shown by applying integration by parts (see Section 3.3 in 2 for similar results).

Lemma 2.8. For all the forms we have consistency and adjoint consistency. That is, if $L u=f$ or $L^{*} w=f$, with $u, w \in H_{0}^{1}(\Omega)$, then

$$
B(u, v)=(f, v) \text { or } B(v, w)=(f, v), \quad \forall v \in V_{h} .
$$

Until now, we have not addressed if (2.2) is well defined. Coerciveness, of course, will not hold for a general second-order elliptic operator. However, using techniques similar to those in Section 4.2 of [2], and in addition taking care of the lower-order terms, we can show the following lemma.

Lemma 2.9. For all three forms there exists a constant $C>0$ such that $\forall \chi \in V_{h}$

$$
\||\chi|\|_{H_{h}^{1}(\Omega)}^{2} \leq C\left(B(\chi, \chi)+\|\chi\|_{L_{2}(\Omega)}^{2}\right) .
$$

If we use this fact, Lemma 2.8 and use the techniques in [10, we could show that our problem is well defined for sufficiently small $h$. 


\section{Local $H^{1}$ estimates}

We start with our main theorem.

Theorem 3.1. Let $D_{0} \subset D_{d} \subset \Omega$ with $\partial_{<}\left(D_{0}, D_{d}\right)=d>\kappa h$ (where $\kappa>1$ is a sufficiently large fixed constant). Suppose $u$ and $u_{h} \in V_{h}$ satisfy

$$
B\left(u-u_{h}, v\right)=0, \quad \forall v \in V_{h}
$$

for any of the forms above. Then,

$\left.||\left|u-u_{h}\right|\right|_{H_{h}^{1}\left(D_{0}\right)} \leq C \inf _{\chi \in V_{h}}\left(|| u-\chi||_{H_{h}^{1}\left(D_{d}\right)}+d^{-1}|| u-\chi \|_{L_{2}\left(D_{d}\right)}\right)+C d^{-1}|| u-u_{h} \|_{L_{2}\left(D_{d}\right)}$, where $C$ is independent of $d$.

Proof. Since $u-u_{h}=(u-\chi)-\left(u_{h}-\chi\right)$, it suffices to show

$$
\left\|\left.\left|u-u_{h}\right|\right|_{H_{h}^{1}\left(D_{0}\right)} \leq C\left(|||u|\left\|_{H_{h}^{1}\left(D_{d}\right)}+d^{-1}|| u\right\|_{L_{2}\left(D_{d}\right)}\right)+C d^{-1}|| u-u_{h}\right\|_{L_{2}\left(D_{d}\right)} .
$$

By the triangle inequality, it will be enough to establish

$$
\left\|\left|u_{h}\right|\right\|_{H_{h}^{1}\left(D_{0}\right)} \leq C\left|\|u \mid\|_{H_{h}^{1}\left(D_{d}\right)}+C d^{-1}\left\|u_{h}\right\|_{L_{2}\left(D_{d}\right)} .\right.
$$

To this end, let $\omega \in C_{<}^{\infty}\left(D_{d / 4}\right)$ with $\omega \equiv 1$ on $D_{d / 8}$ and $|\omega|_{W^{l, \infty}(\Omega)} \leq C d^{-l}$ for $l=0,1, \ldots, r+1$. For a moment, let us assume that we can show the following inequality for all the forms:

$$
\left\|\left|\omega u_{h}\right|\right\|_{H_{h}^{1}(\Omega)}^{2} \leq C B\left(u_{h}, \omega^{2} u_{h}\right)+C d^{-2}\left\|u_{h}\right\|_{L_{2}\left(D_{d}\right)}^{2} .
$$

By (3.1), we can write

$$
B\left(u_{h}, \omega^{2} u_{h}\right)=B\left(u_{h}, \omega^{2} u_{h}-\chi\right)-B\left(u, \omega^{2} u_{h}-\chi\right)+B\left(u, \omega^{2} u_{h}\right)
$$

for any $\chi \in V_{h}$. Since our forms are bounded, we have

$$
\begin{aligned}
B\left(u, \omega^{2} u_{h}\right) & \leq C\left|\left\|u \left|\left\|_{H_{h}^{1}\left(D_{d / 2}\right)}||\left|\omega^{2} u_{h}\right|\right\|_{H_{h}^{1}\left(D_{d / 2}\right)}\right.\right.\right. \\
& \leq C \mid\|u\|_{H_{h}^{1}\left(D_{d}\right)}\left(||\left|\omega u_{h}\right|\left\|_{H_{h}^{1}\left(D_{d}\right)}+d^{-1}|| u_{h}\right\|_{L_{2}\left(D_{d}\right)}\right) .
\end{aligned}
$$

Now, applying the arithmetic-geometric mean inequality, we see that

$$
B\left(u, \omega^{2} u_{h}\right) \leq C\left|\left\|u \left|\left\|_{H_{h}^{1}\left(D_{d}\right)}^{2}+\left.\epsilon||\left|\omega u_{h}\right|\right|_{H_{h}^{1}\left(D_{d}\right)} ^{2}+C d^{-2}\right\| u_{h} \|_{L_{2}\left(D_{d}\right)}^{2} .\right.\right.\right.
$$

Using the boundedness of our forms and Lemma 2.3. we obtain

$$
\begin{aligned}
B\left(u, \omega^{2} u_{h}-\chi\right) & \leq\left.\left. C|\| u|\right|_{H_{h}^{1}\left(D_{d / 2}\right)}||\left|\omega^{2} u_{h}-\chi\right|\right|_{H_{h}^{1}\left(D_{d / 2}\right)} \\
& \leq C \mid\|u\|_{H_{h}^{1}\left(D_{d}\right)}\left(h d^{-1}\left|\omega u_{h}\right|_{H_{h}^{1}\left(D_{d}\right)}+h d^{-2}\left\|u_{h}\right\|_{L_{2}\left(D_{d}\right)}\right) .
\end{aligned}
$$

Taking into account that $h d^{-1}<1$, and applying the arithmetic-geometric mean inequality, we have

$$
B\left(u, \omega^{2} u_{h}-\chi\right) \leq C\left|\left\|u \left|\left\|_{H_{h}^{1}\left(D_{d}\right)}^{2}+\epsilon\left|\omega u_{h}\right|_{H_{h}^{1}\left(D_{d}\right)}^{2}+C d^{-2}\right\| u_{h} \|_{L_{2}\left(D_{d}\right)}^{2} .\right.\right.\right.
$$

Similarly,

$$
B\left(u_{h}, \omega^{2} u_{h}-\chi\right) \leq\left. C h||\left|u_{h}\right|\right|_{H_{h}^{1}\left(D_{d / 2}\right)}\left(d^{-1}\left|\omega u_{h}\right|_{H_{h}^{1}\left(D_{d}\right)}+d^{-2}|| u_{h} \|_{L_{2}\left(D_{d}\right)}\right) .
$$

Using (2.4) and (2.5), we can show the inverse inequality

$$
h\left|\left\|u_{h} \mid\right\|_{H_{h}^{1}\left(D_{d / 2}\right)} \leq C\left\|u_{h}\right\|_{L_{2}\left(D_{d}\right)} .\right.
$$

Therefore, applying the arithmetic-geometric mean inequality one more time gives

$$
B\left(u_{h}, \omega^{2} u_{h}-\chi\right) \leq \epsilon\left|\omega u_{h}\right|_{H_{h}^{1}\left(D_{d}\right)}^{2}+C d^{-2} \|\left. u_{h}\right|_{L_{2}\left(D_{d}\right)} ^{2} .
$$


Finally, using (3.3), (3.4) and making $\epsilon$ small enough in (3.5), (3.6), and (3.7), we arrive at

$$
\|\left.\left|\omega u_{h}\right|\right|_{H_{h}^{1}(\Omega)} ^{2} \leq C\left(\left|\|u \mid\|_{H_{h}^{1}\left(D_{d}\right)}^{2}+d^{-2}\left\|u_{h}\right\|_{L_{2}\left(D_{d}\right)}^{2}\right) .\right.
$$

This will imply (3.2), which in turn implies our theorem.

We are left to show (3.3). We first prove this for the Modified BRMPS form. First, by applying (2.3) and (2.11), we see that

$$
\begin{gathered}
\sum_{e \in \mathcal{E}_{h}} h_{e}\left\|\left\langle\nabla\left(\omega u_{h}\right)\right\rangle\right\|_{L_{2}(e)}^{2}+\sum_{e \in \mathcal{E}_{h}^{0}} h_{e}\left\|\left[\nabla\left(\omega u_{h}\right)\right]\right\|_{L_{2}(e)}^{2} \\
\leq C \sum_{T \in \mathcal{T}_{h}} \int_{T} \nabla\left(\omega u_{h}\right)^{2} d x+C d^{-2}\left\|u_{h}\right\|_{L_{2}(\Omega)}^{2} .
\end{gathered}
$$

By using (3.9), the positive definiteness of $A,(2.19)$ and (2.5), we have

$$
\left\|\left.\left|\omega u_{h}\right|\right|_{H_{h}^{1}(\Omega)} ^{2} \leq C I+C d^{-2}\right\| u_{h} \|_{L_{2}\left(D_{d}\right)}^{2},
$$

where

$$
\begin{aligned}
I \equiv & \sum_{T \in \mathcal{T}_{h}} \int_{T} \nabla\left(\omega u_{h}\right)^{T} A \nabla\left(\omega u_{h}\right) d x+\sum_{e \in \mathcal{E}_{h}} \int_{S_{e}} \omega^{2} r_{A, e}\left(\left[u_{h}\right]\right)^{T} A r_{A, e}\left(\left[u_{h}\right]\right) d x \\
& +\sum_{e \in \mathcal{E}_{h}^{\partial}} \frac{1}{h_{e}} \int_{e} \omega^{2}\left[u_{h}\right]^{2} d s .
\end{aligned}
$$

In the last two inequalities we used the fact that each element has at most $K$ neighbors.

Let

$$
\begin{aligned}
G \equiv & \sum_{T \in \mathcal{T}_{h}} \int_{T} \nabla\left(\omega u_{h}\right)^{T} A \nabla\left(\omega u_{h}\right) d x+2 \sum_{e \in \mathcal{E}_{h}} \int_{S_{e}} \nabla_{h}\left(\omega u_{h}\right)^{T} A \omega r_{A, e}\left(\left[u_{h}\right]\right) d x \\
& +\sum_{e \in \mathcal{E}_{h}} \eta_{e} \int_{S_{e}} \omega^{2} r_{A, e}\left(\left[u_{h}\right]\right)^{T} A r_{A, e}\left(\left[u_{h}\right]\right) d x
\end{aligned}
$$

Using the arithmetic-geometric mean inequality on the middle term and the fact that each triangle has at most $\mathrm{K}$ neighbors, we get

$$
\begin{aligned}
G \geq & \left(1-\frac{K}{\epsilon_{1}}\right) \sum_{T \in \mathcal{T}_{h}} \int_{T} \nabla\left(\omega u_{h}\right)^{T} A \nabla\left(\omega u_{h}\right) d x \\
& +\left(\eta-\epsilon_{1}\right) \sum_{e \in \mathcal{E}_{h}} \int_{S_{e}} \omega^{2} r_{A, e}\left(\left[u_{h}\right]\right)^{T} A r_{A, e}\left(\left[u_{h}\right]\right) d x .
\end{aligned}
$$

Choosing $\epsilon_{1}$ to satisfy $K<\epsilon_{1}<\eta$, we see that

$$
I \leq C\left(G+\sum_{e \in \mathcal{E}_{h}^{\partial}} \frac{1}{h_{e}} \int_{e} \omega^{2}\left[u_{h}\right]^{2} d s\right) .
$$


Using the definition of $G$ and adding and subtracting the terms of $B\left(u_{h}, \omega^{2} u_{h}\right.$ ) (for the Modified BRMPS form) to the right-hand side of (3.11), we arrive at

$$
\begin{aligned}
I \leq & C\left(B\left(u_{h}, \omega^{2} u_{h}\right)\right. \\
& +\sum_{T \in \mathcal{T}_{h}} \int_{T} \nabla\left(\omega u_{h}\right)^{T} A \nabla\left(\omega u_{h}\right)-\nabla\left(\omega^{2} u_{h}\right)^{T} A \nabla u_{h} d x \\
& +\left\{\sum _ { e \in \mathcal { E } _ { h } } \left(2 \int_{S_{e}} \nabla_{h}\left(\omega u_{h}\right)^{T} A \omega r_{A, e}\left(\left[u_{h}\right]\right) d x\right.\right. \\
& \left.\left.+\int_{e}\left(\left\langle\nabla_{h}\left(\omega^{2} u_{h}\right)\right\rangle^{T} A\left[u_{h}\right]+\left\langle\nabla_{h} u_{h}\right\rangle^{T} A\left[\omega^{2} u_{h}\right]\right) d s\right)\right\} \\
& +\sum_{e \in \mathcal{E}_{h}} \eta_{e} \int_{S_{e}}\left(\omega^{2} r_{A, e}\left(\left[u_{h}\right]\right)^{T} A r_{A, e}\left(\left[u_{h}\right]\right)-r_{A, e}\left(\omega^{2}\left[u_{h}\right]\right)^{T} A r_{A, e}\left(\left[u_{h}\right]\right)\right) d x \\
& \left.-\sum_{T \in \mathcal{T}_{h}} \int_{T}\left(c\left(\omega u_{h}\right)^{2}+u_{h} b \cdot \nabla\left(\omega^{2} u_{h}\right) d x+\sum_{e \in \mathcal{E}_{h}} \int_{e}\left\langle u_{h}\right\rangle b \cdot\left[\omega^{2} u_{h}\right] d s\right)\right) \\
= & C\left(B\left(u_{h}, \omega^{2} u_{h}\right)+I_{1}+I_{2}+I_{3}+I_{4}\right) .
\end{aligned}
$$

Note that $I_{4}$ consists of the lower-order terms of our bilinear form. By applying the product rule to each term of $I_{1}$, we see that

$$
I_{1}=\left|\sum_{T \in \mathcal{T}_{h}} \int_{T} u_{h}^{2} \nabla \omega^{T} A \nabla \omega d x\right| \leq C d^{-2}|| u_{h} \|_{L_{2}\left(D_{d}\right)}^{2}
$$

where we also used that $|\nabla \omega|_{L_{\infty}(\Omega)} \leq C d^{-1}$ in the last inequality.

For $I_{2}$, we use the definitions of $P_{A},(2.14)$ and of our lifting operator $r_{A, e}$, to rewrite it as

$$
\begin{aligned}
I_{2}= & -\left(\sum_{e \in \mathcal{E}_{h}} \int_{e} 2\left\langle P_{A}\left(\omega \nabla_{h}\left(\omega u_{h}\right)\right)\right\rangle^{T} A\left[u_{h}\right] d s\right. \\
& \left.-\int_{e}\left(\left\langle\nabla_{h}\left(\omega^{2} u_{h}\right)\right\rangle^{T} A\left[u_{h}\right]+\left\langle\nabla_{h} u_{h}\right\rangle^{T} A\left[\omega^{2} u_{h}\right]\right) d s\right) .
\end{aligned}
$$

By the product rule, $\left\langle\nabla_{h}\left(\omega^{2} u_{h}\right)\right\rangle=\left\langle\omega^{2} \nabla_{h}\left(u_{h}\right)+2 \omega \nabla(\omega) u_{h}\right\rangle$. Adding a term to this and applying the product one more time, we see that

$$
\left\langle\nabla_{h}\left(\omega^{2} u_{h}\right)\right\rangle+\left\langle\omega^{2} \nabla_{h}\left(u_{h}\right)\right\rangle=2 \omega\left\langle\nabla_{h}\left(\omega u_{h}\right)\right\rangle .
$$

Therefore, we can express $I_{2}$ in the following form:

$$
I_{2}=-2 \sum_{e \in \mathcal{E}_{h}} \int_{e}\left\langle P_{A}\left(\omega \nabla_{h}\left(\omega u_{h}\right)\right)-\omega \nabla_{h}\left(\omega u_{h}\right)\right\rangle^{T} A\left[u_{h}\right] d s
$$

By the Cauchy-Schwarz inequality, (2.3) and (2.5),

$$
\begin{aligned}
I_{2} \leq & C \sum_{e \in \mathcal{E}_{h}} h^{-1 / 2}\left\|u_{h}\right\|_{L_{2}\left(S_{e}\right)}\left(h^{-1 / 2} \| P_{A}\left(\omega \nabla_{h}\left(\omega u_{h}\right)\right)\right. \\
& \left.-\omega \nabla_{h}\left(\omega u_{h}\right)\left\|_{L_{2}\left(S_{e}\right)}+h^{1 / 2}\right\| P_{A}\left(\omega \nabla_{h}\left(\omega u_{h}\right)\right)-\omega \nabla_{h}\left(\omega u_{h}\right) \|_{H_{h}^{1}\left(S_{e}\right)}\right) .
\end{aligned}
$$

Moreover, by Lemma 2.4 and (2.10),

$$
I_{2} \leq C \sum_{e \in \mathcal{E}_{h}}\left\|u_{h}\right\|_{L_{2}\left(S_{e}\right)}\left(d^{-1}\left\|\nabla_{h}\left(\omega u_{h}\right)\right\|_{L_{2}\left(S_{e}\right)}+d^{-2}\left\|u_{h}\right\|_{L_{2}\left(S_{e}\right)}\right) .
$$


Finally, using the positive definiteness of $A$ and the arithmetic-geometric mean inequality, we see that

$$
I_{2} \leq \epsilon \sum_{T \in \mathcal{T}_{h}} \int_{T} \nabla\left(\omega u_{h}\right)^{T} A \nabla\left(\omega u_{h}\right) d x+C d^{-2}\left\|u_{h}\right\|_{L_{2}\left(D_{d}\right)} .
$$

Now we bound $I_{3}$. Again, using the definition of $P_{A}$ and $r_{A, e}$, we get that

$$
I_{3}=-\sum_{e \in \mathcal{E}_{h}} \eta_{e} \int_{e}\left\langle P_{A}\left(\omega^{2} r_{A, e}\left(\left[u_{h}\right]\right)\right)-\omega^{2} r_{A, e}\left(\left[u_{h}\right]\right)\right\rangle^{T} A\left[u_{h}\right] d s .
$$

After using (2.5), (2.3), Lemma 2.4 and (2.9), we have

$$
I_{3} \leq C \sum_{e \in \mathcal{E}_{h}}\left\|u_{h}\right\|_{L_{2}\left(S_{e} \cap D_{d}\right)}\left(d^{-1}|| \omega r_{A, e}\left(\left[u_{h}\right]\right)\left\|_{L_{2}\left(S_{e}\right)}+d^{-2} h\right\| r_{A, e}\left(\left[u_{h}\right]\right) \|_{L_{2}\left(S_{e}\right)}\right) .
$$

By Lemma 2.5 and (2.5),

$$
h\left\|r_{A, e}\left(\left[u_{h}\right]\right)\right\|_{L_{2}\left(S_{e}\right)} \leq C\left\|u_{h}\right\|_{L_{2}\left(S_{e}\right)} .
$$

Therefore, after applying the arithmetic-geometric mean inequality, we get

$$
I_{3} \leq C d^{-2}\left\|u_{h}\right\|_{L_{2}\left(D_{d}\right)}^{2}+\epsilon \sum_{e \in \mathcal{E}_{h}} \int_{S_{e}} \omega^{2} r_{A, e}\left(\left[u_{h}\right]\right)^{T} A r_{A, e}\left(\left[u_{h}\right]\right) d x .
$$

Now we handle $I_{4}$. By applying the product rule and the Cauchy-Schwarz inequality, using the boundedness of $c$ and $b$, the positive definiteness of $A$ and (2.5), we have that

$$
\begin{aligned}
I_{4} \leq & C d^{-1}\left\|u_{h}\right\|_{L_{2}\left(D_{d}\right)}^{2}+\epsilon \sum_{T \in \mathcal{T}_{h}} \int_{T} \nabla\left(\omega u_{h}\right)^{T} A \nabla\left(\omega u_{h}\right) d x \\
& +\epsilon \sum_{e \in \mathcal{E}_{h}} \frac{1}{h_{e}} \int_{e}\left(\omega\left[u_{h}\right]\right)^{T} A\left(\omega\left[u_{h}\right]\right) d s
\end{aligned}
$$

If we apply Lemma 2.7 followed by (2.5), we see that

$$
\begin{aligned}
I_{4} \leq & C d^{-2}\left\|u_{h}\right\|_{L_{2}\left(D_{d}\right)}^{2}+\epsilon \sum_{T \in \mathcal{T}_{h}} \int_{T} \nabla\left(\omega u_{h}\right)^{T} A \nabla\left(\omega u_{h}\right) d x \\
& +C_{1} \epsilon \sum_{e \in \mathcal{E}_{h}} \int_{S_{e}} \omega^{2} r_{A, e}\left(\left[u_{h}\right]\right)^{T} A r_{A, e}\left(\left[u_{h}\right]\right) d x,
\end{aligned}
$$

where $C_{1}$ does not depend on $\epsilon$. Finally, by taking $\epsilon$ small enough to "kick back" we arrive at

$$
I \leq C B\left(u_{h}, \omega^{2} u_{h}\right)+C d^{-2}\left\|u_{h}\right\|_{L_{2}\left(D_{d}\right)} .
$$

Therefore, (3.3) holds for the Modified BRMPS form.

In order to work with the LDG form, we define $R(v)=r_{A}([v])+l_{A}\left(\beta^{T}[v]\right)$. Let us assume, for a moment, the following inequality:

$$
\begin{array}{r}
\sum_{e \in \mathcal{E}_{h}} \int_{S_{e}} \omega^{2} r_{A, e}\left(\left[u_{h}\right]\right)^{T} A r_{A, e}\left(\left[u_{h}\right]\right) d x+\sum_{e \in \mathcal{E}_{h}^{0}} \int_{e} \omega^{2} l_{A, e}\left(\beta^{T}\left[u_{h}\right]\right)^{T} A l_{A, e}\left(\beta^{T}\left[u_{h}\right]\right) d x \\
\leq C \sum_{e \in \mathcal{E}_{h}} \frac{1}{h_{e}} \int_{e} \omega^{2}\left[u_{h}\right]^{2} d s+C d^{-2}\left\|u_{h}\right\|_{L_{2}\left(D_{d}\right)} .
\end{array}
$$


Using this inequality together with the fact that

$$
R\left(u_{h}\right)=\sum_{e} r_{A, e}\left(\left[u_{h}\right]\right)+\sum_{e \in \mathcal{E}_{h}^{0}} l_{A, e}\left(\beta^{T}\left[u_{h}\right]\right)
$$

and that $r_{A, e}$ and $l_{A, e}$ are both supported in $S_{e}$, we have

$$
\int_{\Omega} \omega^{2} R\left(u_{h}\right)^{T} A R\left(u_{h}\right) d x \leq C \sum_{e \in \mathcal{E}_{h}} \frac{1}{h_{e}} \int_{e} \omega^{2}\left[u_{h}\right]^{2} d s+C d^{-2}\left\|u_{h}\right\|_{L_{2}\left(D_{d}\right)} .
$$

Using (3.9), we get

$$
\left\|\mid \omega u_{h}\right\|_{H_{h}^{1}(\Omega)}^{2} \leq C J+C d^{-2}\left\|u_{h}\right\|_{L_{2}\left(D_{d}\right)}^{2},
$$

where

$$
J \equiv \sum_{T \in \mathcal{T}_{h}} \int_{T} \nabla\left(\omega u_{h}\right)^{T} A \nabla\left(\omega u_{h}\right) d x+\sum_{e \in \mathcal{E}_{h}} \frac{1}{h_{e}} \int_{e}\left[\omega u_{h}\right]^{2} d s .
$$

Recall that for the LDG form $\eta>0$. Using this fact, the arithmetic-geometric mean inequality and (3.16) we have (see (4.16) in 2] for a similar inequality)

$$
\begin{aligned}
J \leq & C\left(\sum_{T \in \mathcal{T}_{h}} \int_{T} \nabla\left(\omega u_{h}\right)^{T} A \nabla\left(\omega u_{h}\right) d x+2 \sum_{T \in \mathcal{T}_{h}} \int_{T} \omega R\left(u_{h}\right)^{T} A \nabla\left(\omega u_{h}\right) d x\right. \\
& \left.+\int_{\Omega} \omega^{2} R\left(u_{h}\right)^{T} A R\left(u_{h}\right) d x+\sum_{e \in \mathcal{E}_{h}} \frac{\eta_{e}}{h_{e}} \int_{e}\left[\omega u_{h}\right]^{2} d s\right)+C d^{-2}\left\|u_{h}\right\|_{L_{2}\left(D_{d}\right)} .
\end{aligned}
$$

Adding and subtracting the terms of $B\left(u_{h}, \omega^{2} u_{h}\right)$ from the right-hand side of (3.17) we see that

$$
J \leq C B\left(u_{h}, \omega^{2} u_{h}\right)+C\left(J_{1}+J_{2}+J_{3}+J_{4}\right)+C d^{-2}\left\|u_{h}\right\|_{L_{2}\left(D_{d}\right)},
$$

where

$$
\begin{aligned}
& J_{1}=\mid \sum_{T \in \mathcal{T}_{h}} \int_{T} \nabla\left(\omega u_{h}\right)^{T} A \nabla\left(\omega u_{h}\right)-\nabla\left(\omega^{2} u_{h}\right)^{T} A \nabla u_{h} d x \mid, \\
& J_{2}= \mid 2 \sum_{T \in \mathcal{T}_{h}} \int_{T} \omega R\left(u_{h}\right)^{T} A \nabla\left(\omega u_{h}\right) d x \\
&+\sum_{e \in \mathcal{E}_{h}} \int_{e}\left(\left\langle A \nabla_{h}\left(\omega^{2} u_{h}\right)\right\rangle\left[u_{h}\right]\right)+\left\langle A \nabla_{h} u_{h}\right\rangle\left[\omega^{2} u_{h}\right] d s \\
&+\sum_{e \in \mathcal{E}_{h}^{0}} \int_{e}\left(\left[A \nabla_{h}\left(\omega^{2} u_{h}\right)\right] \beta^{T}\left[u_{h}\right]\right)+\left[A \nabla_{h} u_{h}\right] \beta^{T}\left[\omega^{2} u_{h}\right] d s \mid, \\
& J_{3}=\left|\int_{\Omega} \omega^{2} R\left(u_{h}\right)^{T} A R\left(u_{h}\right) d x-\int_{\Omega} R\left(\omega^{2} u_{h}\right)^{T} A R\left(u_{h}\right) d x\right|,
\end{aligned}
$$

and

$$
J_{4}=\left|\sum_{T \in \mathcal{T}_{h}} \int_{T}\left(c\left(\omega u_{h}\right)^{2}+u_{h} b \cdot \nabla\left(\omega^{2} u_{h}\right) d x+\sum_{e \in \mathcal{E}_{h}} \int_{e}\left\langle u_{h}\right\rangle b \cdot\left[\omega^{2} u_{h}\right] d s\right)\right| .
$$

We see that $J_{1}$ is $\left|I_{1}\right|$ with $u_{h}$ now being the LDG solution. Therefore,

$$
J_{1} \leq C d^{-2}\left\|u_{h}\right\|_{L_{2}\left(D_{d}\right)}^{2} .
$$


After following similar manipulations as was done to simplify $I_{2}$, we get

$$
\begin{aligned}
J_{2}= & \mid 2 \sum_{e \in \mathcal{E}_{h}} \int_{e}\left\langle P_{A}\left(\omega \nabla_{h}\left(\omega u_{h}\right)-\omega \nabla_{h}\left(\omega u_{h}\right)\right\rangle^{T} A\left[u_{h}\right] d s\right. \\
& +2 \sum_{e \in \mathcal{E}_{h}^{0}} \int_{e}\left[A\left(P_{A}\left(\omega \nabla_{h}\left(\omega u_{h}\right)\right)-\omega \nabla_{h}\left(\omega u_{h}\right)\right)\right] \beta^{T}\left[u_{h}\right] d s \mid .
\end{aligned}
$$

Using, (2.3), (2.5), Lemma 2.4, and (2.10), we have

$$
J_{2} \leq \epsilon \sum_{T \in \mathcal{T}_{h}} \int_{T} \nabla\left(\omega u_{h}\right)^{T} A \nabla\left(\omega u_{h}\right) d x+C d^{-2}\left\|u_{h}\right\|_{L_{2}\left(D_{d}\right)} .
$$

Using the definitions of our lifting operators and the projection operator, as was done for $I_{3}$, we see that

$$
\begin{aligned}
J_{3}= & \mid \sum_{e \in \mathcal{E}_{h}} \int_{e}\left\langle P_{A}\left(\omega^{2} R\left(u_{h}\right)\right)-\omega^{2} R\left(u_{h}\right)\right\rangle^{T} A\left[u_{h}\right] d s \\
& +\sum_{e \in \mathcal{E}_{h}^{0}} \int_{e}\left[A\left(P_{A}\left(\omega^{2} R\left(u_{h}\right)\right)-\omega^{2} R\left(u_{h}\right)\right)\right] \beta^{T}\left[u_{h}\right] d s \mid .
\end{aligned}
$$

Using the Cauchy-Schwarz inequality, (2.3), and (2.5), we have

$$
\begin{aligned}
J_{3} \leq C \sum_{e \in \mathcal{E}_{h}} h^{-1 / 2}\left\|u_{h}\right\|_{L_{2}\left(S_{e}\right)}\left(h^{-1 / 2} \| P_{A}\left(\omega^{2} R\left(u_{h}\right)\right)\right. & \\
& \left.\quad-\omega^{2} R\left(u_{h}\right)\left\|_{L_{2}\left(S_{e}\right)}+h^{1 / 2}\right\| P_{A}\left(\omega^{2} R\left(u_{h}\right)\right)-\omega^{2} R\left(u_{h}\right) \|_{H_{h}^{1}\left(S_{e}\right)}\right) .
\end{aligned}
$$

Using the fact that $P_{A}$ is linear, the definition of $R$, and the fact that the supports of $r_{A, e}, l_{A, e}$ lie in $S_{e}$, we have

$$
\begin{aligned}
J_{3} \leq & C \sum_{e \in \mathcal{E}_{h}} h^{-1 / 2}\left\|u_{h}\right\|_{L_{2}\left(S_{e}^{\prime}\right)}\left(h^{-1 / 2}\left\|P_{A}\left(\omega^{2} r_{A, e}\left(\left[u_{h}\right]\right)\right)-\omega^{2} r_{A, e}\left(\left[u_{h}\right]\right)\right\|_{L_{2}\left(S_{e}\right)}\right. \\
& \left.\quad+h^{1 / 2}\left\|P_{A}\left(\omega^{2} r_{A, e}\left(\left[u_{h}\right]\right)\right)-\omega^{2} r_{A, e}\left(\left[u_{h}\right]\right)\right\|_{H_{h}^{1}\left(S_{e}\right)}\right) \\
+ & C \sum_{e \in \mathcal{E}_{h}^{0}} h^{-1 / 2}\left\|u_{h}\right\|_{L_{2}\left(S_{e}^{\prime}\right)}\left(h^{-1 / 2}\left\|P_{A}\left(\omega^{2} l_{A, e}\left(\beta^{T}\left[u_{h}\right]\right)\right)-\omega^{2} l_{A, e}\left(\beta^{T}\left[u_{h}\right]\right)\right\|_{L_{2}\left(S_{e}\right)}\right. \\
& \left.+h^{1 / 2}\left\|P_{A}\left(\omega^{2} l_{A, e}\left(\beta^{T}\left[u_{h}\right]\right)\right)-\omega^{2} l_{A, e}\left(\beta^{T}\left[u_{h}\right]\right)\right\|_{H_{h}^{1}\left(S_{e}\right)}\right) .
\end{aligned}
$$

Here $S_{e}^{\prime}$ denotes $S_{e}$ union with the triangles that share an edge with $S_{e}$. Finally, mimicking the argument for bounding $I_{3}$, we see that

$$
\begin{aligned}
J_{3} \leq & C d^{-2}\left\|u_{h}\right\|_{L_{2}\left(D_{d}\right)}^{2}+\epsilon \sum_{e \in \mathcal{E}_{h}} \int_{S_{e}}\left(\omega^{2} r_{A, e}\left(\left[u_{h}\right]\right)^{T} A r_{A, e}\left(\left[u_{h}\right]\right) d x\right. \\
& \left.+\epsilon \sum_{e \in \mathcal{E}_{h}^{0}} \int_{S_{e}} \omega^{2} l_{e}\left(\beta^{T}\left[u_{h}\right]\right)^{T} A l_{e}\left(\beta^{T}\left[u_{h}\right]\right)\right) d x .
\end{aligned}
$$

Furthermore, by (3.15),

$$
J_{3} \leq C d^{-2}\left\|u_{h}\right\|_{L_{2}\left(D_{d}\right)}^{2}+C_{1} \epsilon \sum_{e} \frac{1}{h_{e}} \int_{e}\left[\omega u_{h}\right]^{2} d s,
$$


where $C_{1}$ is independent of $\epsilon$. Again, since $J_{4}$ is $\left|I_{4}\right|$ with $u_{h}$ now being the LDG solution, we have

$$
\begin{aligned}
J_{4} \equiv I_{4} & \leq C d^{-2}\left\|u_{h}\right\|_{L_{2}\left(D_{d}\right)}^{2} \\
& +\epsilon \sum_{T \in \mathcal{T}_{h}} \int_{T} \nabla\left(\omega u_{h}\right)^{T} A \nabla\left(\omega u_{h}\right) d x+\epsilon \sum_{e \in \mathcal{E}_{h}} \frac{1}{h_{e}} \int_{e}\left[\omega u_{h}\right]^{2} d s .
\end{aligned}
$$

Finally, taking $\epsilon$ sufficiently small to "kick back", we conclude that

$$
J \leq C B\left(u_{h}, \omega^{2} u_{h}\right)+C d^{-2}\left\|u_{h}\right\|_{L_{2}\left(D_{d}\right)}^{2} .
$$

This, of course, implies (3.3) for the LDG form, which in turn implies our theorem for the LDG form. We are left to show (3.15).

In bounding $I_{3}$, we showed that

$$
\begin{aligned}
& \left|\sum_{e \in \mathcal{E}_{h}} \int_{S_{e}}\left(\omega^{2} r_{A, e}\left(\left[u_{h}\right]\right)^{T} A r_{A, e}\left(\left[u_{h}\right]\right)-r_{A, e}\left(\omega^{2}\left[u_{h}\right]\right)^{T} A r_{A, e}\left(\left[u_{h}\right]\right)\right) d x\right| \\
& \quad \leq C d^{-2}|| u_{h} \|_{L_{2}\left(D_{d}\right)}^{2}+\epsilon \sum_{e \in \mathcal{E}_{h}} \int_{S_{e}} \omega^{2}\left(r_{A, e}\left(\left[u_{h}\right]\right)\right)^{T} A\left(r_{A, e}\left(\left[u_{h}\right]\right)\right) d x
\end{aligned}
$$

where $\epsilon>0$ is arbitrary. Following similar steps one can show

$$
\begin{gathered}
\left|\sum_{e \in \mathcal{E}_{h}^{0}} \int_{S_{e}}\left(\omega^{2} l_{A, e}\left(\beta^{T}\left[u_{h}\right]\right)^{T} A l_{A, e}\left(\beta^{T}\left[u_{h}\right]\right)-l_{A, e}\left(\omega^{2} \beta^{T}\left[u_{h}\right]\right)^{T} A l_{A, e}\left(\beta^{T}\left[u_{h}\right]\right)\right) d x\right| \\
\leq C d^{-2}\left\|u_{h}\right\|_{L_{2}\left(D_{d}\right)}^{2}+\epsilon \sum_{e \in \mathcal{E}_{h}^{0}} \int_{S_{e}} \omega^{2}\left(l_{A, e}\left(\beta^{T}\left[u_{h}\right]\right)\right)^{T} A\left(l_{A, e}\left(\beta^{T}\left[u_{h}\right]\right)\right) d x
\end{gathered}
$$

By an inverse estimate, (2.5), and the arithmetic-geometric mean inequality, we have

$$
\begin{aligned}
& \left|\sum_{e \in \mathcal{E}_{h}} \int_{S_{e}} r_{A, e}\left(\omega^{2}\left[u_{h}\right]\right)^{T} A r_{A, e}\left(\left[u_{h}\right]\right) d x\right| \\
& \quad+\left|\sum_{e \in \mathcal{E}_{h}^{0}} \int_{S_{e}} l_{A, e}\left(\omega^{2} \beta^{T}\left[u_{h}\right]\right)^{T} A l_{A, e}\left(\beta^{T}\left[u_{h}\right]\right) d x\right| \\
& =\left|\sum_{e \in \mathcal{E}_{h}} \int_{e} \omega\left[u_{h}\right]^{T} A\left\langle r_{A, e}\left(\left[u_{h}\right]\right)\right\rangle d s\right|+\left|\sum_{e \in \mathcal{E}_{h}^{0}} \int_{e} \omega \beta^{T}\left[u_{h}\right]\left[\omega A l_{A, e}\left(\beta^{T}\left[u_{h}\right]\right)\right] d s\right| \\
& \leq C \sum_{e \in \mathcal{E}_{h}} \frac{1}{h_{e}} \int\left[\omega u_{h}\right]^{2} d s+\epsilon \sum_{e \in \mathcal{E}_{h}} \int_{S_{e}} \omega^{2} r_{A, e}\left(\left[u_{h}\right]\right)^{T} A r_{A, e}\left(\left[u_{h}\right]\right) d x \\
& \quad+\epsilon \sum_{e \in \mathcal{E}_{h}^{0}} \int_{S_{e}} \omega^{2} l_{A, e}\left(\beta^{T}\left[u_{h}\right]\right)^{T} A l_{A, e}\left(\beta^{T}\left[u_{h}\right]\right) d x .
\end{aligned}
$$

Using (3.19), (3.20), (3.21), the triangle inequality, and taking $\epsilon$ sufficiently small to "kick back", we arrive at (3.15).

Following similar techniques as those shown above, we can show (3.3) for the Modified BMMPR form. We omit the proof. 
Using approximation properties and Theorem 3.1, we have the following corollary.

Corollary 3.2. Let $x, D_{0}, D_{d}, u$, and $u_{h}$ be as in Theorem 3.1. Then

$$
\|\left.\left|u-u_{h}\right|\right|_{H_{h}^{1}\left(D_{0}\right)} \leq C h^{r-1}|u|_{H^{r}\left(D_{d}\right)}+C d^{-1}|| u-\left.u_{h}\right|_{L_{2}\left(D_{d}\right)} .
$$

\section{Pointwise estimates}

Now we are ready to state our pointwise error estimates.

Theorem 4.1. Suppose $u \in W^{1, \infty}(\Omega)$ satisfies (2.1) and $u_{h} \in V_{h}$ satisfies (2.2) for any of the bilinear forms. Let $x \in \bar{\Omega}$ and $s$ satisfy $0 \leq s \leq r-2, r \geq 2$. Then, there exists a constant $C$ independent of $x, u, u_{h}$ and $h$ such that

$$
\left|\left(u-u_{h}\right)(x)\right| \leq\left. C h \log (1 / h)^{\bar{s}} \inf _{\chi \in V_{h}}|||u-\chi|\right|_{W_{h}^{1, \infty}(\Omega), x, s},
$$

where $\bar{s}=0$ if $0 \leq s<r-2$, and $\bar{s}=1$ if $s=r-2$.

Proof. Now that we have established Lemma 2.6 and Corollary 3.2, we can follow the techniques of the proof for Theorem 5.1 in [6]. The main difference is that their result does not handle hanging nodes. However, their proof can easily be modified to allow hanging nodes by using the fact that each element has at most $K$ neighbors. Also, the norm appearing on the right-hand side of our result has an extra term, but that term can be handled easily throughout their proof.

Similarly, by following the proof of Theorem 5.2 in [6], we obtain gradient pointwise estimates.

Theorem 4.2. Suppose $u \in W^{1, \infty}(\Omega)$ satisfies (2.1) and $u_{h} \in V_{h}$ satisfies (2.2) for any of the bilinear forms. Let $x \in \bar{\Omega}$ and $s$ satisfy $0 \leq s \leq r-1, r \geq 2$. Then, there exists a constant $C$ independent of $x, u, u_{h}$ and $h$ such that

$$
\left|\nabla_{h}\left(u-u_{h}\right)(x)\right| \leq\left. C \log (1 / h)^{\bar{s}} \inf _{\chi \in V_{h}}|||u-\chi|\right|_{W_{h}^{1, \infty}(\Omega), x, s},
$$

where $\overline{\bar{s}}=0$ if $0 \leq s<r-1$, and $\overline{\bar{s}}=1$ if $s=r-1$.

The results here carry over to higher dimensions, if we allow meshes that fit the boundary exactly. Of course, this is not practical in higher dimensions, but it can in principle be done. Let $\Omega^{\prime}$ satisfy $\Omega \subset \Omega^{\prime}$ and $\operatorname{dist}\left(\partial \Omega, \partial \Omega^{\prime}\right) \leq C h^{2}$. If we mesh the larger domain $\Omega^{\prime}$ with simplices such that the simplicial domian contains $\Omega$ and then restrict the mesh to $\Omega$, we will find our desired mesh.

\section{ACKNOWLEDGMENTS}

The author would like to thank Lars Wahlbin and Alfred Schatz for many valuable discussions. The author would also like to thank the referees for their helpful suggestions which lead to a better paper.

\section{REFERENCES}

1. D.N. Arnold, An interior penalty finite element method with discontinuous elements, SIAM J. Numer. Anal., 19(1982), 742-760. MR0664882 (83f:65173)

2. D.N. Arnold, F. Brezzi, B. Cockburn, and L.D. Marini, Unified analysis of discontinuous Galerkin methods for elliptic problems, SIAM J. Numer. Anal., 39(2002), 1749-1779. MR:1885715 (2002k:65183) 
3. F. Bassi, S. Rebay, G. Marrioti, S. Pendinotti, and M. Savini, A high-order accurate discontinuous finite element method for inviscid and viscuous turbomachinery flows, In Proceedings of 2nd European Conference on Turbomachinery, Fluid Dynamics and Thermodynamics (R. Decuypere and G. Dibelius, eds.), Technologisch Instituut, Antwerpen, Belgium, 1997, 99-108.

4. F. Brezzi, G. Manzini, D. Marini, P. Pietra, and A. Russo, Discontinuous Galerkin approximations for elliptic problems, Numer. Methods Partial Differential Equations, 16(2000), 365-378. MR:1765651 (2001e:65178)

5. H. Chen, Local error estimates of mixed discontinuous Galerkin methods for elliptic problems, J. Numer. Math., 12(2004), 1-22. MR2039367 (2005a:65118)

6. H. Chen and Z. Chen, Pointwise estimates of discontinuous Galerkin methods with penalty for second order elliptic problems, SIAM J. Numer. Anal., 42(2004), 1146-1166. MR2113680

7. J. Douglas, Jr., T. Dupont and L. Wahlbin, The stability in $L^{q}$ of the $L^{2}$-projection into finite element function spaces, Numer. Math., 23(1975), 193-197. MR0383789 (52:4669)

8. G. Kanschat and R. Rannacher, Local error analysis of the interior penalty discontinuous Galerkin method for second order elliptic problems, J. Numer. Math., 10(2003), 249-274. MR.1954085 (2004f:65187)

9. J.A. Nitsche and A.H. Schatz, Interior estimates for Ritz-Galerkin methods, Math. Comp., 28(1974), 937-958. MR0373325 (51:9525)

10. A.H. Schatz, An observation concerning Ritz-Galerkin methods with indefinite bilinear forms, Math. Comp., 28(1974), 959-962. MR0373326(51:9526)

11. A.H. Schatz and L.B. Wahlbin, Interior maximum norm estimates for finite element methods, Math. Comp., 31(1977), 414-442. MR0431753(55:4748)

12. A.H. Schatz, Pointwise error estimates and asymptotic error expansion inequalities for the finite element method on irregular grids: Part I. Global Estimates, Math. Comp., 67 (1998), 877-899. MR1464148 (98j:65082)

13. L.B. Wahlbin, Local behavior in finite element methods, In Handbook of Numerical Analysis, Volume II, 353-522, North-Holland, Amsterdam, 1991. MR.1115238

Center for Applied Mathematics, Cornell University, 657 Rhodes Hall, Ithaca, New YORK 14853

E-mail address: jguzman@cam.cornell.edu 